\title{
Evaluation of Exercise Rehabilitation for Survivors of Intensive Care: Pro- tocol for a Single Blind Randomised Controlled Trial
}

\author{
Linda Denehy ${ }^{*}$, , Sue Berney ${ }^{2}$, Elizabeth Skinner ${ }^{2}$, Lara Edbrooke ${ }^{1}$, Stephen Warrillow ${ }^{3}$, \\ Graeme Hawthorne ${ }^{4}$ and Meg E. Morris ${ }^{1}$
}

${ }^{1}$ School of Physiotherapy, The University of Melbourne, Australia

${ }^{2}$ Physiotherapy Department, Austin Health, Melbourne, Australia

${ }^{3}$ Department of Intensive Care, Austin Health, Melbourne, Australia

${ }^{4}$ Department of Psychiatry, The University of Melbourne, Australia

\begin{abstract}
Introduction: Intensive care (ICU) survivors have poor quality of life (QoL) and physical outcomes compared to people of the same age and sex [1]. Although rehabilitation of patients in ICU is now advocated as routine clinical practice [2] there are few randomised controlled trials (RCTs) examining patient outcomes. The primary aim of this single blind randomised controlled trial is to investigate the efficacy of a comprehensive rehabilitation intervention begun in ICU compared to standard care on health related quality of life (HRQoL) and physical function.
\end{abstract}

Method: Participants who have been in one tertiary ICU for $\geq 5$ days, aged $\geq 18$ years, who can understand written and spoken English, will be randomly allocated to receive either standard care or a comprehensive physiotherapy rehabilitation program beginning in ICU and continuing upon discharge to the ward and as an out patient (OP). Blinded assessment of the primary outcome measures physical function, health status and HRQoL will be performed at baseline, 3, 6 and 12 months after discharge. Physical function measures will be obtained at ICU and hospital discharge and for the intervention group, pre and post OP classes. The intervention will include individualised exercises prescribed by physiotherapists in ICU and on the ward and given by an exercise physiologist and physiotherapist in OP.

Results: We aim to enrol 200 participants over two years. The study will determine whether comprehensive physiotherapy rehabilitation from ICU to discharge and OP attendance will improve physical functioning, health status and quality of life in critical care survivors. The cost utility (CUA) and cost effectiveness of such a program will also be evaluated using utility scores and a purpose designed economics questionnaire. Secondary outcomes related to proxy and subject HRQoL comparisons, mechanical ventilation, critical illness neuromyopathy, ICU readmission and discharge destination will also be examined.

Conclusion: The outcomes measured are of significance to critical care patients. The CUA of the intervention will be of interest to health service providers. The results will enable development of clinical practice guidelines for the appropriate exercises in survivors of ICU.

Registered with the Australian and New Zealand Clinical Trials Network [ACTRN 12605000776606].

\section{INTRODUCTION}

Enhanced life saving technologies in Intensive Care Units (ICU) have significantly reduced mortality over the past two decades [1-4]. Improved life saving ability in conjunction with external factors, such as rapid population ageing in Western societies, has increased the demand for Intensive Care [5]. Intensive care services can be expensive for many hospitals and the cost of ICU has recently been estimated at $0.56 \%$ of GDP in the United States [6]. People who remain in ICU for prolonged periods of time ( $>10-14$ days) disproportionately consume resources. In Australia, for example, the average daily cost of an ICU bed is AUD\$2500 $-\$ 3000$ per day and this comprises $20 \%$ of hospital expenditure [7].

*Address correspondence to this author at the School of Physiotherapy, The University of Melbourne, Australia; Tel: 613 83446428;

Fax: 613 83444188; E-mail: 1.denehy@unimelb.edu.au
Previous ICU evaluations have mainly focused on mortality as an outcome. There has been less focus on the health related quality of life (HRQoL) of ICU survivors [8]. Where high rates of survival are achieved, optimising good quality of life should be a goal of management [5, 9-12]. It can be argued that interventions need to be developed that not only improve survival, yet also improve the HRQoL of these survivors. There are few intervention studies where the HRQoL and physical function of ICU survivors have been reported or an economic evaluation carried out, particularly in Australia.

Several observational studies have shown poor physical outcomes and HRQoL in ICU survivors [1, 13-15]. Usually the physical performance of survivors is most affected [11, 16]. At six and 12 months follow up, health status measured using the SF36V2 is lower in patients compared with the population norm $[1,17]$. 
A possible contributor to poor outcomes is that critically ill patients can experience muscle weakness and deconditioning. These may result from prolonged periods of inactivity, catabolism and drug administration (e.g. sedative and neuromuscular blocking agents, corticosteroids) and can last for months post ICU discharge [18]. Severe and prolonged weakness is present in up to $25 \%$ of patients mechanically ventilated for greater than seven days [19]. This ICU acquired weakness is also an independent predictor of prolonged weaning from mechanical ventilation $[20,21]$. Acquired ICU weakness may last for months post ICU discharge [18]. This may impact on physical function of survivors of ICU and may be associated with poor HRQoL post discharge.

Physiotherapy is integral to patient management in the ICU of hospitals in developed countries [19, 22, 23]. Current research is directed towards the acute respiratory management of patients. This shows improved short term physiological outcomes when compared with no treatment or positioning alone [24-27]. The assessment and management of neurological and musculoskeletal complications and the provision of exercise are other important components of the physiotherapist's role [23, 28].

Exercise in ICU aims to mobilise patients early and facilitate weaning from mechanical ventilation [29], optimise oxygenation [30], and improve function by increasing strength and endurance [31]. There is no reliable and valid exercise outcome measure to aid exercise prescription and measure early patient outcomes, making research in the area difficult. As a result, there are currently no exercise guidelines for rehabilitation of ventilated patients and few trials investigating the effect of physiotherapy rehabilitation upon function [18, 32-34]. As far as we are aware there are no published economic evaluations of physiotherapy rehabilitation in ICU.

Studies are therefore needed that evaluate the longer term outcomes for ICU survivors, particularly physical function, HRQoL and economic analyses using specifically designed exercise programs from early hospital admission to beyond discharge $[2,35]$. Thus the primary aims of this trial are to:

Investigate the outcomes of an intensive early ICU, hospital and outpatient exercise intervention compared to standard care on HRQoL and physical function in patients with an ICU length of stay (LOS) $\geq 5$ days

(2) Establish the HRQoL and physical function of Australian critical care survivors up to 12 months after ICU discharge and to

(3) Evaluate the cost effectiveness (CUA) of the exercise intervention as a treatment for ICU survivors.

\section{Research Hypotheses for the Primary Aims}

(H1) Patients who undergo intensive rehabilitation, when compared with standard care, will have improved physical function and HRQoL.

(H2) Improvements in HRQoL will be maintained for 12 months following the comprehensive physiotherapy rehabilitation program.
(H3) The physiotherapy intervention will be more cost effective than standard care.

The secondary aim is to investigate the effect of an early ICU exercise intervention on the duration of mechanical ventilation, length of ICU stay and rate of ICU re-admission.

The study will yield useful information based upon these secondary outcomes but apriori hypotheses have not been formulated as there is insufficient research available upon which to base the hypotheses for these aims.

\section{MATERIALS AND METHODS}

This single blinded randomised controlled trial (RCT) at Austin Health in Melbourne, Australia, will evaluate a comprehensive physiotherapy rehabilitation intervention in survivors of ICU compared with current standard care including a 12 month follow up. Health status is measured using the Short form 36 version 2 (SF36v2) [36] and health related quality of life (HRQoL) using the Assessment of Quality of life and utility instrument (AQoL), [37]. Physical function is measured using the 'PFIT' test [38], six minute walk test (6 MWT, [39]), timed up and go test (TUG, [40]) and physical functioning subscale of the SF36v2. Quality-adjusted life years (QALYS) will be calculated from the SF36V2 using Brazier et al's SF6D [41] and also the AQoL. Costs will be assessed using a purpose-designed health economics questionnaire and a costutility analysis (CUA) and cost effectiveness analysis (CEA) conducted alongside the clinical trial.

\section{PARTICIPANTS}

\section{Sample Size}

Based on the primary outcome of physical function measured using the 6 minute walk test, using preliminary results from [42] $(n=54)$ but reducing improvement between groups to $50 \mathrm{~m}$ (from $80 \mathrm{~m}$ ) with a standard deviation of $110 \mathrm{~m}$, the required sample is 152 . Six months post ICU discharge is the time point used for this sample size calculation. Based on the primary outcome of HRQoL as assessed by the AQoL, the critical effect size is greater than 0.13 utilities [43]. Using alpha $=0.05$ and power $=0.80$, and an effect size of 0.2 in AQoL, the required sample size is 160 cases [44]. Allowing for $20 \%$ mortality [45] for the Austin Hospital ICU Melbourne Australia from the period January 2004 to August 2005 and $15 \%$ from drop outs the total number of participants in the sample size will be increased to 200 if necessary. Over 2 years of recruitment this number of participants is feasible since the Austin Hospital is a level 3 ICU with 20 beds and has an estimated 250 patients per year who stay five days or more in the ICU [45].

\section{Inclusion and Exclusion Criteria}

Two hundred participants in the ICU at Austin Health in Melbourne, Australia and meeting the inclusion criteria will be recruited.

Inclusion criteria: i) age $\geq 18$ years, ii) ICU length of stay $\geq 5$ days, iii) able to understand written and spoken English, iv) reside in greater Melbourne defined as within a radius of $50 \mathrm{~km}$ from Austin Health in Heidelberg and v) the intensive care specialist agrees to their participation. An ICU length of stay $\geq 5$ days was deemed to represent patients with a 'prolonged ICU stay'. This was defined as double the average 
length of stay for ICU survivors in a recent 18 month period (2.5 days) at Austin Health, Melbourne. Patients will be excluded if they i) have major disorders which affect the central nervous system resulting in permanent weakness that is not responsive to exercise training for example neurosurgical intervention, spinal-cord injury (these patients have existing tailored multidisciplinary rehabilitation programmes), ii) have conditions that would render participation in exercise hazardous, such as unstable fractures, iii) are assessed by medical staff as approaching imminent death or withdrawal of medical management is being considered within 48 hours, iv) have an ICU length of stay greater than 5 days due solely to the lack of a suitable general ward bed and v) would have been unable to perform the study physical outcome measures pre-morbidly due to a condition impairing their mobility.

\section{Recruitment and Randomisation}

Patients meeting the above criteria will be asked to participate. Written informed consent from the patient or their next of kin will be sought. If the next of kin provides initial consent, the participant will be asked to consent once they are able. Information regarding screening including the numbers of participants screened and the reasons for exclusion will be recorded following CONSORT guidelines [46].

Within strata, participants will be randomly assigned to receive either standard care plus comprehensive rehabilitation or standard care alone. Participants are not informed about their group allocation, however, once asked to return to out patient exercise classes after discharge the group allocation may be deduced. The strata are diagnosis classification (medical/surgical) and mechanical ventilation at day five (Yes/No), giving four possible randomisation groups. A statistician from the Department of Mathematics and Statistics at The University of Melbourne will perform the randomisation by creation of a random numbers table and use of colour coded (for stratification) opaque numbered envelopes. The intervention physiotherapist will receive an appropriate coloured envelope from the physiotherapy administrative officer once they report on the diagnosis and mechanical ventilation status of the participant. A third independent person will confirm the envelope matches the stratification group necessary prior to opening. Once opened the group allocation and envelope number are recorded and the envelope stored.

\section{PROCEDURE}

The Human Research Ethics Committee of Austin Health approved the study and the protocol is registered with the Australian and New Zealand Clinical Trials Registry ACTRN 12605000776606.

The planned flow of participants through the study is given in Fig. (1). Once consent is obtained participants will be referred to a physiotherapist who will administer the HRQoL questionnaires to the patient and/or the patient proxy (next of kin) before randomisation. If the patient is awake they will also perform the baseline 'PFIT' test. Participants will then be randomly assigned to receive the intervention or standard care.

\section{Standard Care}

Both groups will receive usual medical, physiotherapy and nursing care in the ICU ward and upon discharge home. Standard physiotherapy care in the ICU involves assessment and treatment of the respiratory system and may include positioning, hyperinflation techniques and suctioning [35]. The physical function of the patient is assessed and active bed exercises and mobility are encouraged as soon as possible and may include sitting out of bed, marching on the spot or ambulating. In the ward functional activities are encouraged, with an emphasis on mobility. Participants may participate in functional maintenance exercise classes. Any participants may be transferred to a rehabilitation facility if deemed necessary as per standard care. The ward physiotherapist is involved in discharge planning for all participants.

\section{Physiotherapy Rehabilitation (Intervention)}

The intervention group will receive an intensive rehabilitation protocol commencing during their ICU stay. Once participants fulfil the inclusion criteria and are safe to exercise according to defined criteria (refer to Table 1) they will commence the intensive rehabilitation program in the ICU. The intervention protocol will consist of an exercise regimen based on performance of the baseline 'PFIT' test. Exercises will include sitting out of bed, sit to stand exercise with or without assistance, marching on the spot and active shoulder elevation. These exercises are consistent with regimens used by Australian physiotherapists as reported in pilot data obtained from an Australia wide survey [38] undertaken prior to study commencement. For participants unable to sit out of bed or stand up, upper and lower limb active and active assisted exercises will be performed. The use of a tilt table or standing frame may be employed. Treatment in the ICU will involve exercise for up to a total of fifteen minutes per day for each participant. Exercise will increase to twice daily once subjects are able to be disconnected from the ventilator for $>4$ hours or until they are considered to be successfully weaned from mechanical ventilation. Weaning from mechanical ventilation will proceed according to a standardised unit weaning protocol. Once discharged to the general ward the physiotherapist will continue and progress the intensive rehabilitation program. Exercise will be progressed according to performance on the $6 \mathrm{MWT}$, calculating 6 minute walk work or if able, a submaximal $\mathrm{VO}_{2}$ test undertaken on a stationary cycle using a standardised protocol [47].

The physiotherapist will treat each participant twice daily aiming to exercise for $30 \mathrm{~min}$ at each session with gradual progression toward one $60 \mathrm{~min}$ exercise session until discharge from hospital. Upon discharge home participants will be booked to commence their outpatient program. This will be offered for eight weeks with the aim of attending two one hour classes per week out of the three offered. Exercise at home in the form of a walking program will be encouraged on days not attending the program and a printed laminated instruction sheet provided. Out patient programs will be commenced within two weeks of the participant being discharged from hospital. Programs will be individualized by the physiotherapist but run in an exercise class structure. Ward and outpatient exercise classes will comprise functional, resistance and aerobic components with intensity set using the modified Borg scale [48]. Exercise prescription will be based upon a further submaximal cycle test or 6 MWT.

\section{OUTCOME MEASURES}

Baseline descriptive data collection will include age, sex, body mass index, smoking and social history, diagnosis, 


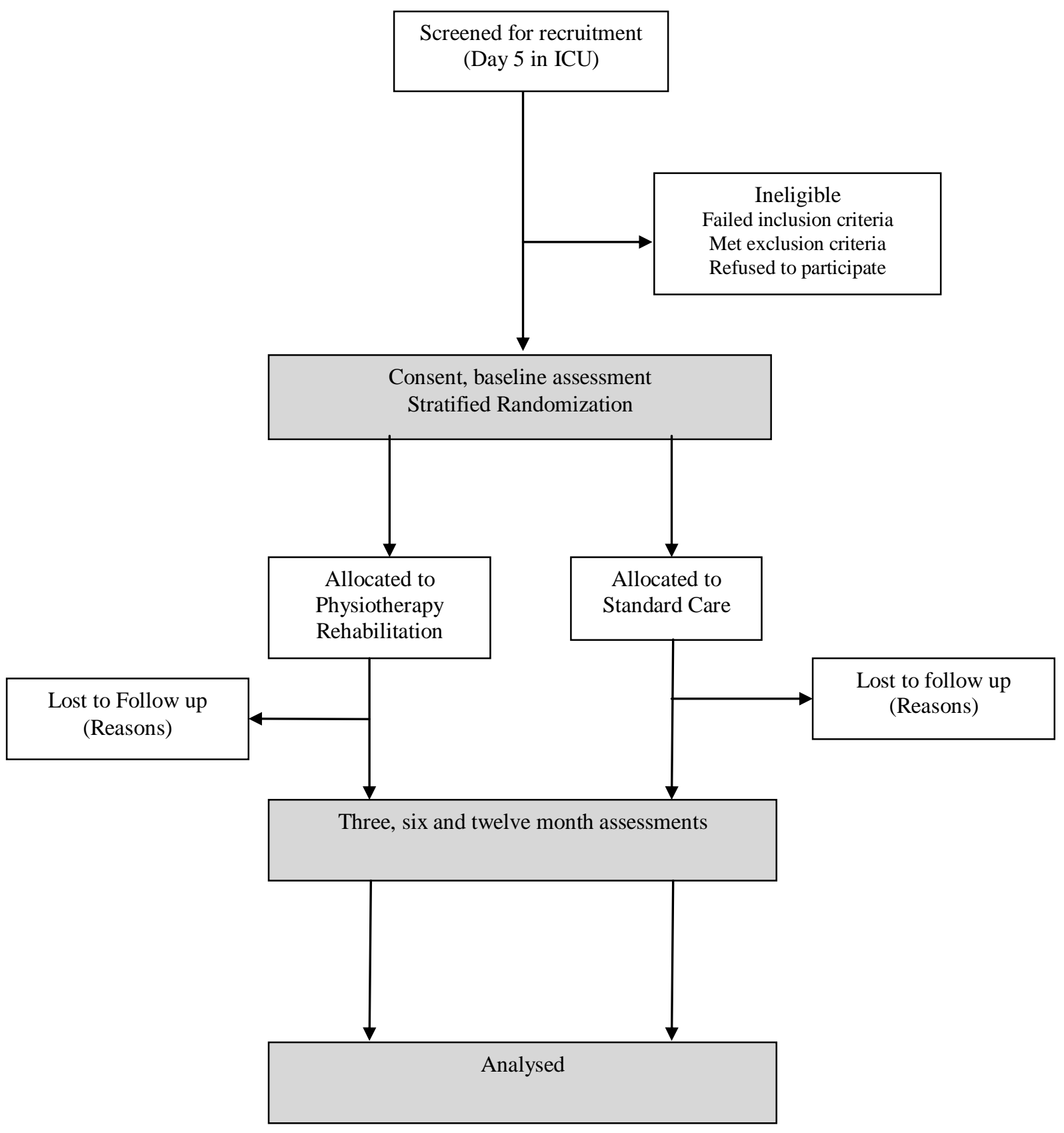

Fig. (1). Participant enrolment and retention flow diagram.

Acute Physiological Chronic Health Evaluation (APACHE II) score and date of admission to ICU. Mechanical ventilation hours, length of ICU stay (days/hours), incidence of ICU readmission, discharge destination and survival will be also be recorded.

The primary outcome measures are physical function and HRQoL. All primary outcome measurements will be undertaken at baseline, 3, 6 and 12 months after ICU discharge. Research assistants blinded to group allocation will perform all outcome assessments. The success of blinding will be assessed using a short outcome assessor questionnaire for each time point and the number of 'un-blinding' occasions will be recorded. This is deemed necessary as complete blinding in a clinical environment is often difficult to achieve. Physical function measures will also be performed upon ICU discharge and hospital discharge and for the intervention group additionally pre and post out patient classes.

\section{Health Status}

The Australian version of the SF36v2 [36] will be used to measure health status. It comprises 36 items which provide health status profile scores in eight dimensions (Physical 
function, Role physical, Bodily pain, General health, Vitality, Social function, Role emotion and Mental health). Unlike the SF36V1, the SF36V2 is more sensitive in those items assessing problems with work or daily activities because the dichotomous response categories for these items have been replaced with 5-point response scales. It also differs from the SF36V1 in that scores are presented as Tscores (mean $50, \mathrm{SD}=10$ ). For a full discussion of the differences between the international and Australian versions of the SF36V2 including Australian norms and scoring weights the reader should consult Hawthorne et al. (2007) [49]. The clinimetric properties of the SF36V1 have been well established on samples from diverse populations, including studies in critical care populations $[14,50]$. The reliability coefficients for SF36 in 166 adult patients in ICU were reported to be greater than 0.75 [50]. Construct and content validity were also reported to be acceptable.

\section{Table 1. Safety Guidelines for Exercise Sessions}

\section{Safety Guidelines: Exercise Should Not be Delivered or Should be Ceased When:}

1. Patient mean arterial blood pressure $(\mathrm{MABP})<65 \mathrm{mmHg}$ or $>$ $120 \mathrm{mmHg}$ or $\leq 10 \mathrm{mmHg}$ lower than normal systolic or diastolic in renal patients

2. Patient heart rate is $<50$ or $>140$ beats/minute and/or a new arrhythmia developed (including frequent ventricular ectopics or new onset atrial fibrillation)

3. Patient requiring $>5 \mu \mathrm{g} /$ minute noradrenaline (or equivalent vasopressor/inotrope)

4. $\mathrm{FiO}_{2}>0.6$ with $\mathrm{PaO}_{2}<70 \mathrm{~mm} \mathrm{Hg}$

5. $\mathrm{PEEP}>8 \mathrm{~cm} \mathrm{H}_{2} \mathrm{O}$

6. Patient suffers a $10 \%$ oxygen desaturation below their resting $\mathrm{SaO}_{2}$ or if $\mathrm{SaO}_{2}<85 \%$.

7. Patient respiratory rate $>35$ breaths $/$ minute

8. Patient temperature $>38$ degrees

9. Patient not to exercise if they have suffered an acute clinical deterioration (eg. requiring reintubation) or an acute bout of sepsis.

10. Patient is drowsy, unco-operative or unable to follow commands/instructions

11. Patient suffers a fall during transfer to the chair or walking

12. Patient became pale and sweaty and/or the patient specifically requested to stop due to feeling acutely unwell

13. Patient complained of new onset chest pain

\section{HRQoL and Utility}

The AQoL instrument is a multi-attribute utility instrument comprising 15 items in 5 dimensions. The first three items which assess health care resource consumption are not used in computing the utility score. The life-death utility scale score range is from 1.00 (best possible HRQoL equivalent state), to 0.00 (death equivalent state) and -0.04 (worse than death equivalent state) [37]. AQoL utility scores will be compared with Australian population norms [43]. The AQoL utility score was designed to allow the calculation of QALYs for use in a cost-utility analysis, which will be conducted alongside the clinical trial. The AQoL has undergone exten- sive validation in different populations, but has not been previously used in ICU survivors. The minimal important difference in utility is reported to be 0.06 [43].

The SF6D, which uses 12 items from the SF36v2, will also be used to provide utilities. The SF6D life-death scale score range is from 1.00 (full health) to 0.30 (worst health state measured by the SF6D) [41]. Recent research shows that there are theoretical difficulties with the SF6D utilities leading to very different utility estimates than those provided by the AQoL [51].

Both the AQoL and SF6D estimates will be used in the CUA, thus comparing the two most recently developed utility measures in a longitudinal study. The SF6D has not to our knowledge been utilised previously in ICU survivors.

Health status and HRQoL data will be collected at baseline, 3, 6 and 12 month follow up. Several studies report the need for a long term follow up in this patient population [5, $52,53]$. At baseline, the questionnaires will be completed by a proxy (next of kin) and the participant where possible. At all other time points the data will be collected from both participants and proxies because while the literature shows that proxy assessments are moderately correlated (kappa $>0.6$ ) with patient assessments $[54,55]$ it is not well researched how proxy and patient assessments vary in relation to each other over time. One of the few studies to report this found that agreement was highest for pre-surgery and 6-months discharge and worse at 12-month follow-up [56].

\section{Economic Evaluation Incorporating a CU Study}

A health economist from the School of Population Health at The University of Melbourne will advise the study team on the economic evaluation, specifically in relation to assignment of health service data collection, costs and the CUA. Based on this advice the perspective of the study will be societal, as recommended by the International Panel on Cost-Effectiveness [57]. All health service delivery costs (including intervention costs, such as the training of physiotherapists) associated with the ICU will be collected. These include clinician, nursing and other ICU staff, direct treatment medical aides and theatre, pharmaceutical and hospital stay costs. Follow up treatment costs will also be collected. Use of services (including intervention services) during this period will be identified through patient records. Nonrecorded service use will be ascertained through patient interview at each of the follow up periods. Where costs are not directly available from the hospital, service use will be costed using annual national benchmark costs published by the Australian Institute of Health and Welfare. In addition, costs will be assigned to patient time receiving treatment, the costs of care giving (both paid and unpaid), and other treatment-related costs (e.g. travel, domestic duties, changes in accommodation, repairs). Wages foregone will be included where the patient was working prior to admission to the ICU.

Four levels of consequences will be assessed: improvements in functional capacity on the physical function tests, changes in health status (the SF36V2), presumed 'costs saved' as a result of the intervention (e.g. lower unplanned re-admissions during the study period), and gains in utility (as measured by the AQoL and SF6D). 
A cost-effectiveness analysis (CEA) of the intervention will be undertaken based on the cost per health unit outcome for functional capacity, health status changes and costs saved. A CUA will also be conducted, based on QALY gains as a result of the intervention, where the QALY gains are derived from utility gains during the follow up period and modelled life expectancy. Two analyses will be conducted and compared, based respectively on the AQoL and the SF6D.

\section{Physical Function}

Will be assessed using several different methods:

'PFIT' test is a test comprising four elements: marching on the spot (MOS), moving from sitting to standing, an upper limb endurance activity and measurement of muscle strength. Each element is rated by recording the time for MOS, the number of assistants needed to stand from sitting, the time taken and number of repetitions that shoulders can be flexed and strength using the score on the Oxford muscle scale [58] for two muscle groups. This test was previously found to be reliable $(\mathrm{ICC}=0.99-1.0)$ and responsive to change $(\mathrm{p}<0.05)$ in our pilot study conducted in 12 participants and will be used to measure function in the ICU [38]. This is a novel test and further development of the test using results from the current study will be undertaken.

(ii) The 6 minute walk test is a commonly used practical and simple test that requires a $25 \mathrm{~m}$ hallway but no specific exercise equipment. It measures the distance that a person can quickly walk on a flat, hard surface in 6 minutes, is self paced and submaximal and reflects the functional exercise level for daily physical activities. It correlates moderately with peak oxygen uptake measured by formal exercise testing $(\mathrm{r}=0.5$ 0.7) [59] in populations with cardiorespiratory impairment. Two tests will be performed to reduce the variability due to practice [60]. The 6-minute walk test has been used in diverse populations, including a recent report in ICU [61].

(iii) The timed up and go test (TUG) records the time taken in seconds for participants to stand from a chair, walk three metres return and sit down again [40]. It is simple to perform and inexpensive, standardised instructions are used and two tests are performed. Values can be compared to normal age related times [62]. It is a test of functional mobility in older adults and has good correlation with gait speed $(r=0.745)$ [63] and the Berg Balance test $(r=0.47)$ [64]. Inability to complete the TUG has been reported to predict hospital length of stay in one recent study [65]. Although the TUG has been validated in older persons the responsiveness of this test has not previously been reported in an ICU population.

\section{PARTICIPANT RETENTION STRATEGIES}

Long term follow up is an important challenge for researchers studying ICU survivors, to reduce the possibility of selection bias resulting from loss to follow up [66].
All participants will be contacted by the trial co-ordinator to make appointments for follow up and standardised protocols for follow up telephone contact with participants will be employed. If participants are unable to travel to the hospital, taxi vouchers, volunteer drivers or parking permits will be offered to help them to attend for classes or outcome measure appointments. A letter will be sent one month prior to follow up appointments to inform participants that telephone contact will be made to make an appointment. Three attempts will be made to contact participants by telephone. If contact is not made then a further letter will be sent asking for participants to contact the trial co-ordinator. The Austin Health Medtrak ${ }^{\circledR}$ system will be checked prior to all follow up to check whether any trial participant has been entered into the system as being re-admitted or deceased. In addition, a quarterly newsletter will be developed and sent to all participants.

\section{SAFETY PROTOCOLS}

All physiotherapists providing intervention in the trial are experienced ICU clinicians. Both therapists involved in the intervention arm of the study and those performing outcome measures will undertake routine safety training including basic life support and calling an emergency code at the Austin Hospital. Written manuals of protocols and safety guidelines will be made available to all research personnel. Criteria for commencing and ceasing exercise or outcome measurement are outlined in Table 1. If any adverse event occurs either during or up to an hour following intervention or outcome measurement e.g. fall or onset of acute illness, the chief investigators will be notified.

Serious adverse events are defined in the trial as "adverse events which result in a medical emergency team (MET) call being made. These events could include: death, a fall resulting in injury, cardiac arrest or stroke. Serious adverse events may require: admission to a critical care area or surgical intervention". These events will also be reported to the Austin health human research ethics committee.

A data monitoring committee will examine trial safety by reviewing any adverse events at regular intervals. Data process indicators, based on the consort diagram, will be presented monthly by research personnel to identify potential safety, recruitment, treatment, drop out rates and follow up contact issues.

\section{DATA MANAGEMENT}

All data will be entered into a purposefully designed Access data base and exported to SPSS version14 for analyses. Descriptive statistics will first be used to examine the distributions for the two groups comparing baseline scores, to describe mean outcomes and to test whether the assumptions for use of parametric tests have been met. Analyses will be by intention to treat and per protocol. Repeated measures analysis of variance (or covariance) with planned comparisons will be used to compare all continuous variables that are normally distributed. Where data are non-normally distributed, log transformation or non-parametric analyses will be conducted. Categorical variables will be analysed using the chi square statistic. Where appropriate, analyses will be reported with mean difference and 95\%CIs. A sensitivity 
analysis will be used with the economic evaluation data and the marginal costs reported.

\section{RESULTS}

We aim to enrol 200 participants over two years. The study will determine whether comprehensive physiotherapy rehabilitation from ICU to discharge and OP attendance will improve physical functioning, health status and quality of life in critical care survivors. The CUA and cost effectiveness of such a program will also be evaluated using utility scores and a purpose designed economics questionnaire. Secondary outcomes related to proxy and subject HRQoL comparisons, mechanical ventilation, critical illness neuromyopathy, ICU readmission and discharge destination will also be examined.

\section{DISCUSSION}

Advances in technology and the application of research outcomes into clinical practice have resulted in improved survival rates for patients in the ICU [4]. The provision of intensive care services is expensive and in the current climate of limited health resources, it is important to provide interventions that not only sustain life but lead to reasonable life quality in ICU survivors. The HRQoL, physical function and long-term outcomes of survivors of ICU has not yet been studied in an intervention trial in Australia, although the international literature reports poor health status and HRQoL outcomes compared to age-matched controls in this heterogeneous population.

This research will be the first intervention study to evaluate the effect of a comprehensive physiotherapy exercise program for patients from ICU through to discharge home. It will also provide information on the cost-effectiveness of such an intervention. The important primary outcomes for the patient are expected to be improved physical function, health status and improved HRQoL. Given the potentially devastating effects of a critical illness on the survivor and their carers [67], gaining improved function and enhanced QoL will be important outcomes for all ICU survivors.

The results will be used to develop guidelines for the rehabilitation of ventilated patients for translation into clinical practice. Currently no specific guidelines except for safety of exercise [68] exist to guide clinicians in the type, intensity or clinical value of exercises during ICU admission. Furthermore, apart from a recent examination of functional maintenance programs for medical patients [69], there is little research relating to the outcomes of application of an intensive exercise program once ICU patients are discharged to the general hospital ward. Having ICU survivors return to an eight week outpatient program after discharge is based upon successful rehabilitation strategies in other cardiorespiratory patient populations [70]. If the study findings are positive and cost effective it is possible that rehabilitation programs may be set up elsewhere. Patients returning for exercise could also potentially be assessed by other health professionals to monitor their HRQoL, physical and psychological functioning, providing an ICU follow up clinic [2].

The use of only one tertiary referral centre is a possible limitation of this study. However, the variability in approach to patient management between ICUs, particularly in application of mechanical ventilation strategies and weaning may impact on this already heterogeneous patient population resulting in difficulties in managing such a project and achieving conclusive outcomes. The ICU at Austin Health, Victoria, Australia is similar in bed size, staffing and patient demographic to other major metropolitan mixed medical/surgical units in Australia (ANZICS data base, 2005). Additionally we utilised the types of exercises that were consistent with those reportedly provided by physiotherapists in our pilot work which surveyed exercise prescription in Australian and New Zealand ICUs.

\section{CONCLUSION}

This single blinded RCT will evaluate an intensive physiotherapy rehabilitation intervention in survivors of ICU compared with current standard care including a 12 month follow up. The strengths of the project are that the outcomes being measured are of significance to the patient cohort and the cost utility of the intervention will be of interest to health service providers. This is important given the limitation of health care resources and the anticipated increased demand from an aging population. The findings will provide the potential to develop future clinical practice guidelines for the provision of exercise in survivors of ICU.

\section{KEY MESSAGES}

- $\quad$ Poor physical outcomes and HRQoL in ICU survivors have been reported

- This study tests the effects of an exercise program commenced in ICU, continued on the general ward and out patients compared with standard care

- Participants in the intervention group will receive graded, individualised strength and endurance exercises, aiming at improvement in function

- $\quad$ Standardised instruments for physical function and HRQoL will be used to measure outcomes at baseline, 3, 6 and 12 months from ICU discharge.

- Cost utility and cost effectiveness analyses will be performed alongside the clinical trial

\section{ACKNOWLEDGEMENTS}

This research is supported by the National Health and Medical Research Council (NHMRC, Australia; Project Grant 454717) and the Physiotherapy Research Foundation, Australia. Preliminary work was undertaken by ES as part of a doctoral qualification. The authors thank the current project staff, Jeremy Friedman, Hwee Ong, Jacki Rowe, Libby Proud and Kimberley Haines for their hard work and the staff of the physiotherapy department at Austin Health, particularly Guy Metcalf, Rob Lopressti and Linda Rautela for their able help in setting up computer networks (GM) and measuring outcomes and Dr David Berlowitz for his input in the development of the PFIT test.

\section{AUTHOR'S CONTRIBUTIONS}

All authors contributed to the study design, method and writing. LD, SB, ES, SW, GH and MM helped to develop the successful grant proposals which support the study. SB and ES contributed the exercise strategies for ICU, ward intervention and novel PFIT test ideas, LE specifically contributed to protocols related to outpatients and participant 
retention strategies. MM and SW were involved in providing advice on safety, monitoring and ethical issues and MM will chair the data monitoring committee. GH advised specifically on HRQoL, sample size calculation and utility analyses and LD drafted the manuscript. All other authors critically revised it for important intellectual content. All authors contributed to and approved the final version of the manuscript for publication.

\section{REFERENCES}

[1] Cuthbertson B, Scott J, Strachan M, Kilonzo M, Vale L. Quality of life before and after intensive care. Anaesthesia 2005; 60(8): 332-9.

[2] Morris P, Herridge M. Early intensive care unit mobility: Future directions. Crit Care Clin 2007; 23(14): 97-110.

[3] Bernard G, Vincent J, Laterre P, et al. Efficacy and safety of recombinant human activated protein $\mathrm{C}$ for severe sepsis. N Engl J Med 2001; 344(11): 699-709.

[4] Van Den Berghe G, Wouters P, Weekers F, et al. Intensive insulin therapy in critically ill patients. N Engl J Med 2001; 345(9): 135967.

[5] Williams T, Dobb G, Finn J, Webb S. Long-term survival from intensive care: A review. Intensive Care Med 2005; 31(10): 130615.

[6] Halpern N, Pastores S, Greenstein R. Critical care medicine in the United States 1985-2000: an analysis of bed numbers, use and costs. Crit Care Med 2004; 32: 1254-9.

[7] Judson J, Fisher M. Intensive care in Australia and New Zealand. Crit Care Clin 2006; 22(17): 407-23.

[8] Rivera-Fernández R, Sánchez-Cruz J, Abizanda-Campos R, Vázquez-Mata G. Quality of life before intensive care unit admission and its influence on resource utilization and mortality rate. Crit Care Med 2001; 29(9): 1701-9.

[9] Flaatten H, Kvale R. Survival and quality of life 12 years after ICU. A comparison with the general norwegian population. Intensive Care Med 2001; 27(7): 1005-11.

[10] Herridge M, Angus D. Acute lung injury - Affecting many lives. N Engl J Med 2005; 353(3): 1736-8.

[11] Herridge M, Cheung A, Tansey C, et al. One-year outcomes in survivors of the acute respiratory distress syndrome. N Engl J Med 2003; 348(11): 683-93.

[12] Keenan SP, Carlet J, Curtis R, Pinsky M, Rubenfeld G. Patient centred outcomes. J Crit Care 2002; 17: 221-34.

[13] Combes A, Costa M, Trouillet J, et al. Morbidity, mortality, and quality-of-life outcomes of patients requiring $>14$ days of mechanical ventilation. Crit Care Med 2003; 31(9): 1375-81.

[14] Dowdy D, Eid M, Sedrakyan A, et al. Quality of life in adult survivors of critical illness: A systematic review of the literature. Intensive Care Med 2005; 31(10): 611-20.

[15] Lizana FG, Bota DP, De Cubber M, Vincent J. Long-term outcome in ICU patients: What about quality of life? Intensive Care Med 2003; 29: 1286-93.

[16] Kaarlola A, Pettilä V, Kekki P. Quality of life six years after intensive care. Intensive Care Med 2003; 29(6): 1294-9.

[17] Elliott D, Mudaliar Y, Kim C. Examining discharge outcomes and health status of critically ill patients: some practical considerations. Intensive Crit Care Nurs 2004; 20: 366-77.

[18] Jones C, Skirrow P, Griffiths R, et al. Rehabilitation after critical illness: A randomized, controlled trial. Crit Care Med 2003; 31(6): 2456-61

[19] De Jonghe B, Sharshar T, Lefaucheur J, et al. Paresis acquired in the intensive care unit: A prospective multicenter study. JAMA 2002; 288(9): 2859-67.

[20] De Jonghe B, Bastuji-Garin S, Sharshar T, Outin H, Brochard L. Does ICU-Acquired paresis lengthen weaning from mechanical ventilation? Intensive Care Med 2004; 30(5): 1117-21.

[21] Garnacho-Montero J, Amaya-Villar R, García-Garmendía J, Madrazo-Osuna J, Ortiz-Leyba C. Effect of critical illness polyneuropathy on the withdrawal from mechanical ventilation and the length of stay in septic patients. Crit Care Med 2005; 33(6): 34954.

[22] Herridge M. Long-term outcomes after critical illness: Past, present, future. Curr Opin Crit Care 2007; 13(3): 407-23.

[23] Stiller K. Physiotherapy in intensive care: Towards an evidencebased practice. Chest 2000; 118(13): 1801-13.
[24] Berney S, Denehy L. The effect of physiotherapy treatment on oxygen consumption and haemodynamics in patients who are critically ill. Aust J Physiother 2003; 49: 99-105.

[25] Berney S, Denehy L, Pretto J. Head down tilt and manual hyperinflation enhance sputum clearance in patients who are intubated and ventilated. Aust J Physiother 2004; 50: 9-14.

[26] Hodgson C, Carroll S, Denehy L. A survey of manual hyperinflation in Australian hospitals. Aust J Physiother 1999; 45: 185-93.

[27] Ntoumenopoulos G, Presneill J, McElholum M, Cade J. Chest physiotherapy for the prevention of ventilator-associated pneumonia. Intensive Care Med 2002; 28: 850-6.

[28] Denehy L. The use of manual hyperinflation in airway clearance. Eur Respir J 1999; 14: 958-65.

[29] Cirio S, Piaggi G, De Mattia E, Nava S. Muscle retraining in ICU patients. Monaldi Arch Chest Dis 2003; 59(4): 300-3.

[30] Dean E, Ross J. Discordance between cardiopulmonary physiology and physical therapy. Toward a rationale basis for practice. Chest 1992; 101: 1694-8.

[31] Jones A. Physiotherapy in intensive care. In: Oh TE, Ed. Intensive care manual. 4th ed: Oxford: Butterworth-Heinemann 1997; 26-32. Clini E, Ambrosino N. Early Physiotherapy in the respiratory Intensive care unit. Respir Med 2005; 99(9): 1096-104.

[33] Martin U, Hincapie L, Nimchuk M, Gaughan J, Criner G. Impact of whole-body rehabilitation in patients receiving chronic mechanical ventilation. Crit Care Med 2005; 33(7): 2259-65.

[34] Nava S. Rehabilitation of patients admitted to a respiratory intensive care unit. Arch Phys Med Rehabil 1998; 79: 849-54.

[35] Denehy L, Berney S. Physiotherapy in the intensive care unit. Phys Ther Rev 2006; 11: 1-8.

[36] Ware J, Kosinski M, Dewey J. How to score version 2 of the SF-36 health survey. Lincoln: Quality Metric Inc 2000

[37] Hawthorne G, Richardson J, Osborne R. The assessment of quality of life (AQoL) instrument: a psychometric measure of health related quality of life. Qual Life Res 1999; 8: 209-24.

[38] Skinner E, Berney S, Warrillow S, Denehy L. Rehabilitation and exercise prescription in Australian intensive care units. Physiotherapy 2008; 94: 220-9.

[39] Nici L, Donner C, Wouters E, et al. Society ATSatER. American thoracic society/European respiratory society statement on pulmonary rehabilitation. Am J Respir Crit Care Med 2006; 173: 1390413.

[40] Podsiadlo D, Richardson S. The timed "Up and Go" test: A test of basic functional mobility for frail elderly persons. J Am Geriatr Soc 1991; 39: 142-8

[41] Brazier J, Roberts J, Deverill M. The estimation of a preferencebased measure of health from the SF-36. J Health Econ 2002; 21 : 271-92.

[42] Burtin C, Clerckx B, Robbeets C, et al. Effectiveness of early exercise in critically ill patients: preliminary results. Intensive Care Med 2006; 32: 109.

[43] Hawthorne G, Osborne R. Population norms and meaningful differences for the Assessment of Quality of Life (AQoL) measure. Aust N Z J Public Health 2005; 29(7): 136-42.

[44] Cohen J. Statistical power analyses for behavioral sciences. $2^{\text {nd }}$ ed New York: Academic Press 1997.

[45] ANZICS 2006. Available from: http://sas.anzics.com.au/portal [cited 2006 21/04/2006].

[46] Moher D, Schulz K, Altman D. The CONSORT statement: Revised recommendations for Improving the quality of reports of parallelgroup randomised trials. Lancet 2001; 357(4): 1191-4.

[47] Selig S, Gosling C, Carlson J. A multi-stage step test protocol for people with low exercise capacity. Clin Kinesiol 2000; 54: 67-71.

[48] Borg G. Ratings of perceived exertion and heart rates during shortterm cycle exercise and their use in a new cycling strength test. Int J Sports Med 1982; 3: 153-8.

[49] Hawthorne G, Osborne RH, Taylor A, Sansoni J. The SF36 version 2: critical analyses of population weights, scoring algorithms and population norms. Qual Life Res 2007; 16: 661-73.

[50] Chrispin PS, Scotton H, Rogers J, Lloyd D, Ridley SA. Short form 36 in the intensive care unit: assessment of acceptability, reliability and validity of the questionnaire. Anaesthesia 1997; 52: 15-23.

[51] Hawthorne G. Measuring incontinence in Australia. Canberra: commonwealth of Australia 2006.

[52] Azoulay E, Kentish-Barnes N, Pochard F. Health-related quality of Life: An outcome variable in critical care survivors. Chest 2008; 133(3): 339-41. 
[53] Dowdy D, Needham D, Mendez-Tellez P, Herridge M, Pronovost P. Studying outcomes of intensive care unit survivors: The role of the cohort study. Intensive Care Med 2005; 31(8): 914-21.

[54] Hofhuis J, Hautvast J, Schrijvers A, Bakker J. Quality of life on admission to the intensive care: Can we query the relatives? Intensive Care Med 2003; 29(6): 974-9.

[55] Rogers J, Ridley S, Chrispin P, Scotton H, Lloyd D. Reliability of the next of kin's estimates of critically ill patients' quality of life. Anaesthesia 1997; 52: 1137-43.

[56] Elliot D, Lazurus R, Leeder S. Proxy respondents reliably assessed the quality of life of elective cardiac surgery patients. J Clin Epidemiol 2006; 59: 153-9.

[57] Manning W. Panel on cost-effectiveness in health and medicine recommendations: identifying costs. J Clin Psychiatry 1999; 60(Suppl 3): 54-8.

[58] Daniels L, Worthingham C. Muscle testing: Techniques of manual examination. Philadelphia: W.B. Saunders; 1995.

[59] Cahalin L, Pappagianopoulos P, Prevost S, Wain J, Ginns L. The relationship of the 6-min walk test to maximal oxygen consumption in transplant candidates with end-stage lung disease. Chest 1995; 108: 452-9.

[60] Jenkins S. 6-Minute walk test in patients with COPD: Clinical applications in pulmonary rehabilitation. Physiotherapy 2007; 93(8): 175-82.

[61] Galle S, Burtin C, Clerckx B, et al. Effectiveness of early exercise in critically ill patients. Acta Anaesthesiol Belg 2007; 58(1): 88 .
[62] Bohannon RW. Reference values for the timed up and go test: A descriptive meta-analysis. J Geriatr Phys Ther 2006; 29: 64-8.

[63] Freter SH, Fruchter N. Relationship between timed 'up and go' and gait time in an elderly orthopaedic rehabilitation population. Clin Rehabil 2000; 14: 96-101.

[64] Bennie S. Measurements of balance: comparison of the Timed "Up and Go" test and functional reach test with the berg balance scale. J Phys Ther 2003; 15: 93-7.

[65] Large J, Gan N, Basic D, Jennings N. Using the timed up and go test to stratify elderly inpatients at risk of falls. Clin Rehabil 2006; 20: 421-8.

[66] Needham D, Dennison C, Dowdy D, et al. Study protocol: The Improving Care of Acute Lung Injury Patients (ICAP) study. Crit Care 2006; 10(1): R9.PMID: 16420652 [available online] http://ccforum.com/content/10/1/R9

[67] Van Pelt D, Milbrandt E, Qin L, et al. Informal caregiver burden among survivors of prolonged mechanical ventilation. Am J Respir Crit Care Med 2007; 175: 167-73.

[68] Stiller K. Safety issues that should be considered when mobilizing critically ill patients. Crit Care Clin 2007; 23: 35-53.

[69] de Morton N, Keating J, Jeffs K. The effect of exercise on outcomes for hospitalised older acute medical patients: an individual patient data meta-analysis. Age Ageing 2007; 36: 219-22.

[70] Nici L, Donner C, Wouters E, et al. American thoracic society/European respiratory society statement on pulmonary rehabilitation. Am J Respir Crit Care Med 2006; 173: 1390-413.

(C) Denehy et al.; Licensee Bentham Open.

This is an open access article licensed under the terms of the Creative Commons Attribution Non-Commercial License (http://creativecommons.org/licenses/by$\mathrm{nc} / 3.0 /$ ) which permits unrestricted, non-commercial use, distribution and reproduction in any medium, provided the work is properly cited. 